\title{
Effect of different treatments and alcohol addiction on gut microbiota in minimal hepatic encephalopathy patients
}

\author{
ZAN ZUO $^{1,2}$, HONG FAN $^{1,2}$, XIAO-DAN TANG ${ }^{2}$, YAN-MIN CHEN $^{2}$, LIN-TING XUN $^{2}$, \\ YAN LI ${ }^{2}$, ZHENG-JI SONG ${ }^{2}$ and HUI-QIONG ZHAI ${ }^{3}$
}

\author{
${ }^{1}$ College of Environmental Science and Engineering, Kunming University of Science and Technology, Kunming, \\ Yunnan 650500; ${ }^{2}$ Department of Gastroenterology, Affiliated Hospital of Kunming University of Science and Technology, \\ The First People's Hospital of Yunnan, Kunming, Yunnan 650532; ${ }^{3}$ Department of Gastroenterology, \\ Yanan Hospital of Kunming, Kunming, Yunnan 650032, P.R. China
}

Received September 8, 2016; Accepted May 15, 2017

DOI 10.3892/etm.2017.5141

\begin{abstract}
Minimal hepatic encephalopathy (MHE) is caused by dysbiosis of gut microbiota, particularly the ammonia-producing bacteria. Given the efficacy of certain treatments on MHE and the connection between alcoholism and MHE, a thorough understanding of how these strategies affect the gut microbiota in patients (alcoholic or non-alcoholic) will facilitate the assessment of their efficacy in the reshaping of gut microbiota. In the present study, a metagenomics approach was adopted to reveal alterations in gut microbiota of 14 MHE patients following treatment with rifaximin alone or rifaximin plus probiotics. Patients were grouped into the alcoholic and non-alcoholic groups to examine differences in terms of their response to treatment. Treatment reduced the overall microbiota diversity and decreased the abundance of certain ammonia-producing bacteria, such as Clostridium, with the treatment of rifaximin plus probiotics presenting a more apparent effect. Non-alcoholic MHE patients responded better to the treatment, as they presented greater reduction in microbiota diversity and a more consistent decline in certain ammonia-producing bacteria genera (such as Clostridium and Streptococcus) belonging to the Firmicutes phylum. In conclusion, treatment with rifaximin alone and rifaximin plus probiotics exhibited a different effect in different MHE patients, decreasing the overall gut microbiota diversity to various extents and reshaping microbiota in different ways. Furthermore, non-alcoholic MHE patients responded better to treatment in microbiota alterations.
\end{abstract}

Correspondence to: Professor Hong Fan, Department of Gastroenterology, Affiliated Hospital of Kunming University of Science and Technology, The First People's Hospital of Yunnan, 157 Jinbi Road, Xishan, Kunming, Yunnan 650532, P.R. China E-mail:kmfanhong@163.com

Key words: gut microbiota, hepatic encephalopathy, rifaximin, probiotics, alcoholic liver cirrhosis

\section{Introduction}

Hepatic encephalopathy (HE), presenting as functional dysregulation in the central nervous system, is a cognitive syndrome induced by liver diseases and characterized by dysbiosis in the metabolism (1). The 1-year survival rate of HE patients has been reported to be $42 \%$, while the 3 -year survival rate declines to $23 \%$ (1). Approximately $30 \%$ of cirrhotic patients develop minimal HE (MHE), which displays no apparent symptoms, but results in a higher risk of developing overt HE and of mortality (2). Therefore, diagnosis and treatment at the MHE stage is of crucial significance in reducing the mortality rates in liver cirrhosis patients. A comprehensive understanding of MHE pathogenesis and associated biomarkers in its diagnosis and treatment is, thus, of increasing importance.

Several hypotheses have been proposed for the pathogenesis of HE, including toxicosis by ammonium, manganese, false neurotransmitters and imbalance in plasma insulin and amino acids (1-6). The patients in the present study were split into alcoholic and non-alcoholic groups because liver cirrhosis may be induced by alcoholism, while certain liver cirrhotic patients develop MHE. MHE can also be caused by various pathological conditions, including an accumulation of mercaptans and short-chain fatty acids (7). Numerous of these hypotheses are associated with dysbiosis in the ammonium metabolism; therefore, ammonium toxicosis is regarded as one of the most important inducing factor of HE (4). Certain urease-producing gut microbes, including Klebsiella, Proteus and Helicobacter pylori, have been demonstrated to be associated with blood ammonium levels and are implicated in the pathogenesis of HE. Therefore, targeting these microbes for therapeutic purposes is of great potential for the treatment of HE. For instance, probiotics and prebiotics are used to lower the gut $\mathrm{pH}$ and prevent the growth of the urease-producing bacteria (8). In addition, MHE has been reported to be a risk factor for motor vehicle accidents due to attention deficit caused by MHE patients (9), while a recent study suggested that yoghurt consumption was an independent negative risk factor for traffic accidents in patients with liver cirrhosis (10). Furthermore, antibiotics are also typically employed to control the proliferation of Helicobacter pylori (11). However, no 
reports currently exist on the effect of combined treatment with antibiotics (such as rifaximin, used in the present study) and probiotics on gut microbiota alterations. Besides, alcoholic and non-alcoholic MHE patients may also respond differently to treatment in terms of gut microbiota alterations. Therefore, investigation into these aspects will reveal how gut microbiota are differentially regulated by different pathogenic causes and therapeutic regimens, and therefore provide guidance for the drugs used to target the specific pathogenic causes.

As an increasing number of patients with chronic hepatitis is reported in the southwestern Yunnan in China, particularly patients infected with hepatitis $\mathrm{C}$ virus, the incidence of liver cirrhosis with or without HE is likely to increase. Given the correlation between the metabolic functions of gut microbiota and $\mathrm{HE}$, the aim of various therapeutic regimens is to restore the gut microbiota towards the normal composition and functions.

Therefore, the present study conducted the first comprehensive metagenomics investigation into gut microbiota alterations subsequent to treatment with rifaximin, a well-documented anti-MHE drug, or a combination treatment consisting of rifaximin and probiotics (12). The comparison of the two treatments investigated in the current study provides a deeper insight into the effect of different treatments on gut microbiota. Furthermore, the study subjects were grouped into alcoholic and non-alcoholic MHE patients, reporting the different responses observed in these patients and therefore providing guidance for the design of more effective treatment regimens.

\section{Patients and methods}

Patients. A total of $14 \mathrm{MHE}$ patients from the Affiliated Hospital of Kunming University of Science and Technology, (Kunming, China) were recruited into the present investigation. The characteristics of these patients are presented in Table I. Liver cirrhotic patients, induced by alcoholism, hepatitis B virus infection or other causes, were recruited initially. These patients were then diagnosed using multiple testing, including number connection test (NCT) and digit symbol test (DST). In the NCT, 25 numbers were distributed on a piece of paper, and patients were required to connect the same numbers within the shortest time possible. Mistakes were corrected in a timely manner and the test continued. The total time required to complete the assessment was recorded. In the DST, a matrix of nine symbols were provided to symbolize the numbers 1-9, and patients were required to fill the corresponding symbols underneath the numbers in the shortest time possible. Scores were calculated according to the number of correct answers and adjusted according to the age range. Scores of $\leq 7$ were considered to be abnormal. Patients with abnormal scores in both NCT and DST were diagnosed as MHE patients and recruited as study subjects. Patients that were diagnosed as alcoholic liver cirrhosis were grouped into the alcoholic category, whereas liver cirrhotic patients that were induced by other factors, including hepatitis $\mathrm{B}$ virus infection, were grouped into the non-alcoholic category.

All procedures were conducted with informed consent from the patients and in consistency with ethical requirement of the ethics board of Affiliated Hospital of Kunming University of Science and Technology.
Treatment. Patients were treated with rifaximin or with rifaximin (Nanjing Pharmaceuticals Co., Ltd., Nanjing, China) and probiotics on a random basis. Rifaximin tablets $(400 \mathrm{mg}$ ) were administered orally twice a day, and samples were collected 4 weeks after the treatment. The probiotic (trade name, Meichangan) formula included live combined Bacillus subtilis and Enterococcus faecium enteric-coated 250-mg capsules (Hanmi Pharmaceutical Co., Ltd., Beijing, China) containing 0.5 billion live bacteria each.

Sample collection. Samples were collected under sterile conditions using sputum collection box. Faeces were flash frozen in liquid nitrogen $2 \mathrm{~h}$ after collection and preserved at $-80^{\circ} \mathrm{C}$. DNA from the intestinal bacteria community of patients was isolated immediately prior to treatment and 4 weeks following treatment with rifaximin or rifaximin plus probiotics, using the CTAB (hexadecyltrimethylammonium bromide) approach (13). Agarose gel electrophoresis $(0.8 \%)$ was performed to assess DNA concentration and purity, followed by dilution to $1 \mathrm{ng} / \mu \mathrm{l}$. Next, DNA samples were subjected to metagenomic analysis in order to obtain information on the changes in the composition of bacterial species following treatment.

Sequencing strategy of bacterial samples. In order to determine the bacterial composition in the subjects' intestines, polymerase chain reaction was employed to amplify the highly variable V3, V4 and V5 regions of bacterial 16S rRNA using specifically designed primers (F515, 5'-GTGCCAGCMGCCGCG GTAA-3' and R806, 5'-GGACTACVSGGGTATCTAAT-3'; $\mathrm{M}$ stands for $\mathrm{A} / \mathrm{C}, \mathrm{V}$ stands for $\mathrm{A} / \mathrm{C} / \mathrm{G}$, and $\mathrm{S}$ stands for $\mathrm{C} / \mathrm{G}$ ) as previously described (14). While in conventional testing only the V4 region of $16 \mathrm{~S}$ rRNA is amplified, the present study investigated more regions, therefore greatly enhancing the sensitivity and specificity of the approach.

DNA was extracted from samples, followed by quality validation, as previously described (14). Qualified DNA was used as a template for the amplification of the V3-V5 region of $16 \mathrm{~S}$ rRNA. Following purification, amplicons were subjected to analysis with MiSeq system according to the manufacturer's instructions (Illumina, Inc., San Diego, CA, USA) to obtain primary sequences. Adapters and low-quality reads were removed from the primary sequences, followed by multiple bioinformatics analysis, including operational taxonomic unit (OTU), abundance, $\alpha$ and $\beta$ diversity, and clustering analyses.

OTU analysis and statistics at various taxonomic levels. Original sequences were filtered, as previously described (14) to remove low-quality reads. Valid reads were subjected to the UCLUST-based clustering methodology in QIIME software (version 2; Scikit-Bio open source; http://qiime.org/). Sequences with $>95 \%$ similarity were clustered as one OTU, denoting one bacterial species. The taxonomic levels included order, family, genus and species. All procedures were completed as previously described (14).

$\alpha$ diversity analysis. $\alpha$ diversity is used to describe the abundance of various species in the sample. Based on OTU analysis, rarefaction curves were generated using $\mathrm{R}$ to demonstrate the observed number of OTUs and the estimated number of species (Chao1 index) with the increase of read numbers (15). Chaol 
Table I. Patient characteristics and treatment.

\begin{tabular}{|c|c|c|c|c|c|c|}
\hline Patient & Sex & Age, years & Cause of cirrhosis & Year of diagnosis & $\mathrm{DST}, \min$ & Treatment \\
\hline 1 & Male & 65 & Autoimmune hepatitis & 2011 & 46 & $\mathrm{RP}$ \\
\hline 2 & Male & 57 & Hepatitis B cirrhosis & 2011 & 34 & $\mathrm{R}$ \\
\hline 3 & Female & 48 & Primary biliary cirrhosis & 2012 & 10 & $\mathrm{R}$ \\
\hline 4 & Female & 55 & Autoimmune hepatitis & 2011 & 13 & $\mathrm{RP}$ \\
\hline 5 & Female & 46 & Hepatitis B cirrhosis & 2010 & 35 & $\mathrm{RP}$ \\
\hline 6 & Male & 48 & Hepatitis B/alcoholic & 2011 & 31 & $\mathrm{RP}$ \\
\hline 7 & Male & 41 & Autoimmune hepatitis & 2006 & 50 & $\mathrm{RP}$ \\
\hline 8 & Female & 43 & Autoimmune hepatitis & 2008 & 50 & $\mathrm{R}$ \\
\hline 9 & Male & 39 & Hepatitis B cirrhosis & 2009 & 21 & $\mathrm{R}$ \\
\hline $10^{\mathrm{a}}$ & Male & 57 & Alcoholic cirrhosis & 2012 & 26 & $\mathrm{RP}$ \\
\hline $11^{\mathrm{a}}$ & Male & 47 & Alcoholic cirrhosis & 2011 & 26 & $\mathrm{R}$ \\
\hline $12^{\mathrm{a}}$ & Male & 60 & Alcoholic cirrhosis & 2011 & 48 & $\mathrm{RP}$ \\
\hline $13^{\mathrm{a}}$ & Male & 68 & Alcoholic cirrhosis & 2007 & 35 & $\mathrm{R}$ \\
\hline $14^{\mathrm{a}}$ & Male & 63 & Alcoholic cirrhosis & 2009 & 43 & $\mathrm{R}$ \\
\hline
\end{tabular}

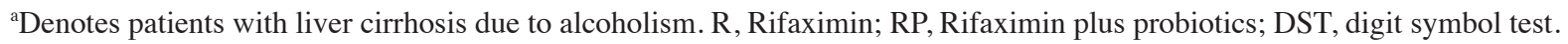

index is used to estimate the number of OTUs or species in the population, therefore symbolizing the diversity of the population. Shannon diversity indices of various samples were also calculated to assess the diversity and distribution evenness of the species. This index differs from others in that it takes into account the distribution evenness of the species. Therefore, these indices can denote the diversity and distribution of the species in a given population. The detailed analytical method was performed as previously described (15).

$\beta$ diversity analysis. $\beta$ diversity, represented by the principal coordinate analysis (PcoA), reveals the magnitude of community composition and describes the alterations in species distribution in various major coordinates. PcoA using weighted Unifrac from the 28 samples calculated the values in three principal components- PC1, PC2 and PC3. PcoA analysis then locates the samples in plots against three principal coordinates to showcase the relative similarities and abundances of the samples. The principal coordinates stand for a matrix of major components, which account for $39.67,22.38$ and $11.99 \%$ of the microbiota composition, respectively. The dot plot provided a direct image of differences in the intestinal microbiota of each subject post-treatment compared with pre-treatment by examining at the distance between these samples. The detailed analytical method was performed as previously described (15).

Clustering analysis. Based on the data of distribution at the phylum and genus level, clustering was conducted using the matrix of unweighted and weighted Unifrac, as previously described (15). Fewer branches present between the two samples indicate that they are closer in species distribution.

\section{Results}

Study patients. A total of 14 MHE patients were recruited in the present investigation. Among these, 7 patients were treated with rifaximin alone, while the remaining 7 were treated with rifaximin in conjugation with probiotics. When grouping according to the alcohol consumption of patients, 9 patients were defined as non-alcoholic MHE patients, whereas the remaining 5 were categorized as alcoholic MHE patients. Table I gives the basic information of the patients and their treatment. Patients prior to treatment were denoted as numbers 1-14 and after treatment, the patients were denoted as the number+treatment. For instance, 1+RP stands for sample from patient 1 after Rifaximin plus Probiotics treatment, whereas $2+\mathrm{R}$ stands for sample from patient 2 after Rifaximin treatment.

Basic data set collection and analysis. In total, 28 samples from the 14 study subjects prior to and following treatment yielded a data set consisting of $1,585,825$ high-quality classifiable $16 \mathrm{~S}$ rRNA gene sequences, with a mean of 56,637 sequences per sample. Using the conventional criterion of $95 \%$ sequence similarity (corresponding to taxonomically valid species), a total of 4,456 OTUs were identified, with a mean of 159 OTUs per sample. These OTUs were divided into 10 genera. The top four genera included Lactobacillus and Bacteroides, which were the two dominant genera, as well as Streptococcus and Clostridium.

Clinical treatment generally reduced gut microbiota diversity in patients. The number of observed species in each sample with the increment in sequence numbers is shown in Fig. 1A. An overall reduction in the actual numbers of observed species was observed post-treatment. For instance, the species number in patient 5 was decreased following treatment with rifaximin. In contrast, certain patients presented the opposite trend, such as patient 8 who had slightly higher species number post rifaximin treatment. The marginal difference in estimated species numbers prior to and following treatment with rifaximin plus probiotics appeared to be smaller compared with that in patients treated with rifaximin alone. 

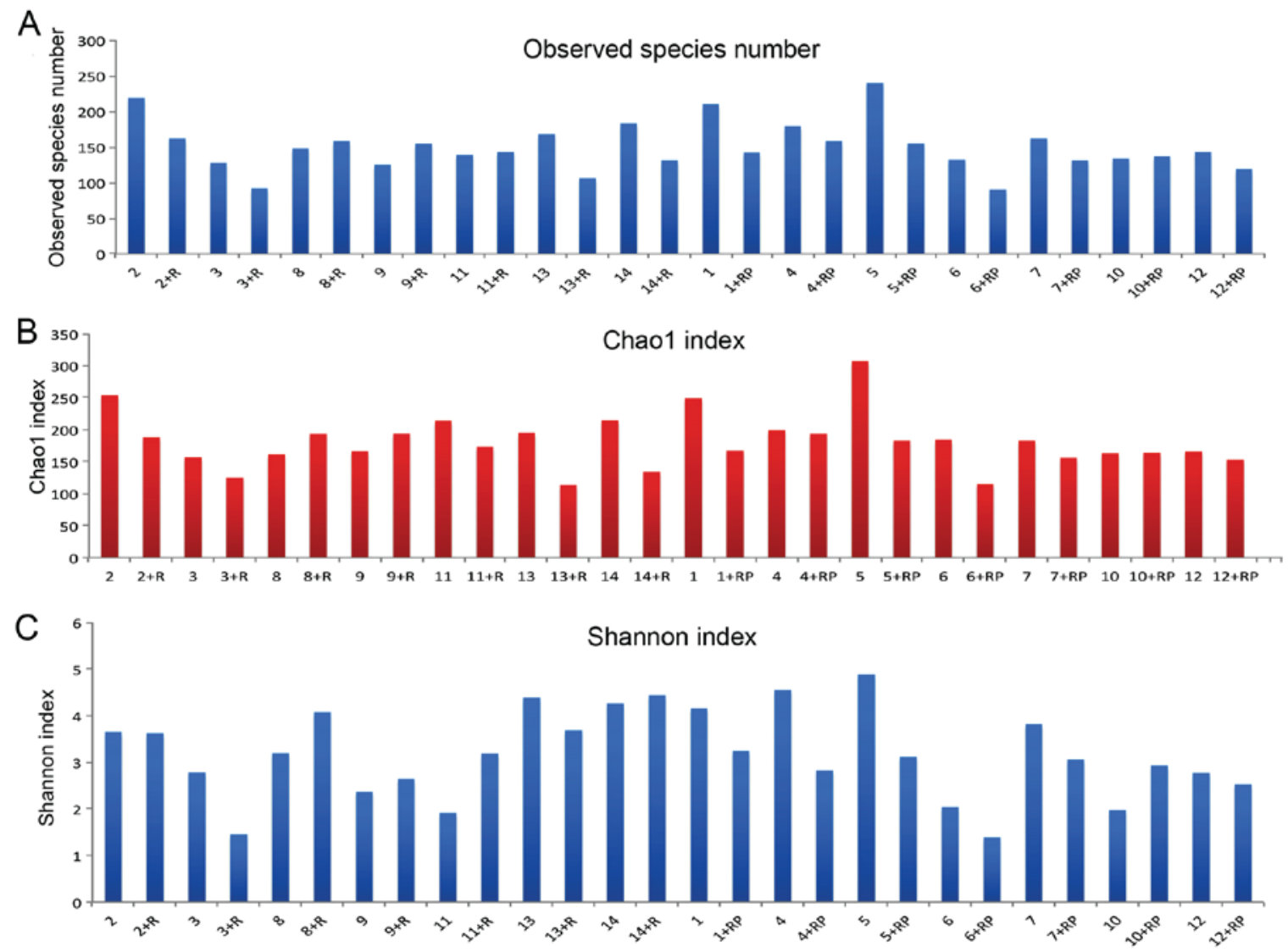

Figure 1. Clinical treatment generally reduced gut microbiota diversity in patients. (A) Observed species number alterations in each sample with the increment in sequence numbers. (B) Chaol index was calculated to estimate the total number of operational taxonomic units based on the actual observed species number. (C) Shannon index alterations in each sample with the increase of sequence numbers.

The Chaol index was calculated to estimate the total number of OTUs based on the actual observed species number. An overall decrease in Chaol index was detected subsequent to treatment, although certain exceptions were observed (Fig. 1B). Changes in Chaol index before and after treatment and the differences in Chaol index between the two treatments are presented in Fig. 1B and roughly corresponded to the trend detected for species numbers (Fig. 1A).

Shannon index is shown in Fig. 1C. By comparing the Shannon index of each patient prior to and following treatment, a general decline in the index was observed following treatment, with a few exceptions, such as patient 10 . Upon taking a closer look, a predominant reduction in the index can be observed following treatment with rifaximin plus probiotics, whereas the group with rifaximin only treatment presented a more diversified response. Furthermore, certain patients, such as patient 3 , presented a reduced Shannon index following rifaximin treatment, while others presented the opposite effect, such as patient 8 . In addition, patient 2 did not have an evident response to rifaximin treatment in terms of microbiota diversity, as Shannon index remained almost the same subsequent to treatment.

Similarly, if subjects are divided into the alcoholic and non-alcoholic MHE groups, a predominant decrease in Shannon index is observed in non-alcoholic patients following treatment. By contrast, alcoholic liver cirrhosis patients demonstrated divergent responses, with patient 11 presenting a higher index, and patients 12 and 13 exhibiting lower values post treatment.
Rank abundance of the samples also revealed reduced diversity following treatment. Rank abundance, presented in Fig. 2, visualizes the species richness and evenness in the sample. The total number of species is demonstrated by the maximum reading of each curve on the $\mathrm{x}$-axis. In general, these maximal $\mathrm{x}$ values in Fig. 2 are higher in samples with higher observed species numbers (Fig. 1A) and higher Chaol index (Fig. 1B). Species evenness, as deduced from the slope of the curves, was generally higher in samples with a higher number and more homogeneous distribution of species.

Alcohol addiction compromises treatment efficacy. Fig. 3A presents a heatmap of the results of $\beta$ diversity analysis with all the weighted (top value in each box) and unweighted (bottom value in each box) Unifrac values between two patients according to pairwise comparison, prior to or following treatment. Comparing the values for each patient prior to and following treatment, the magnitude of response to the treatment for that specific patient is obtained. For instance, the weighted Unifrac for patient 11 pre- and post-rifaximin treatment was 0.133 , demonstrating the least response to the treatment. By contrast, a large difference in microbiota was observed in patient 9, with a weighted UniFrac value of 0.602 . Furthermore, no notable difference was observed between the two groups receiving different treatment. However, non-alcoholic MHE patients displayed generally higher Unifrac values as compared with alcoholic MHE patients.

Weighted PcoA analysis calculates the values in three principal components-PC1, PC2 and PC 3 . It then locates 


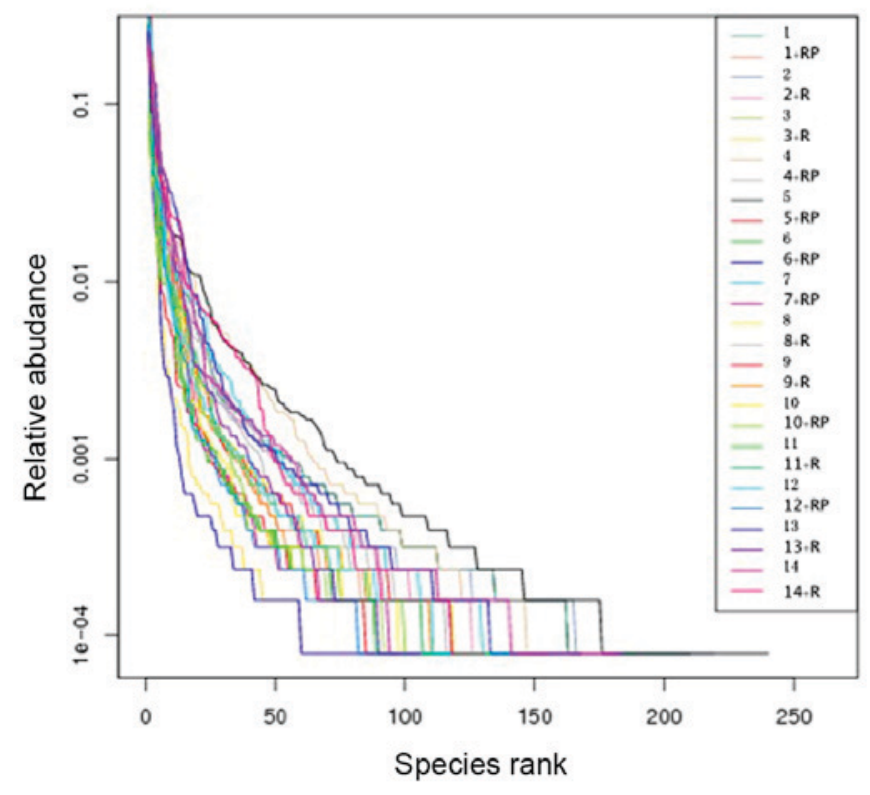

Figure 2. Rank abundance, demonstrating the species richness and evenness in the sample, also revealed reduced microbiota diversity after treatment. The total number of species is shown in the maximum reading of each curve on the $\mathrm{x}$-axis, while values on the $\mathrm{y}$-axis demonstrate the relative abundance of each ranked species.

the samples in plots against the three principal coordinates to showcase the relative similarities and abundances of the samples. The principal coordinates stand for a matrix of major components, which account for 39.67, 22.38 and $11.99 \%$ of the microbiota composition, respectively (Fig. 3B-D). It was also observed that non-alcoholic MHE patients presented better clustering in these principal coordinates (PC1, PC2 and PC3; with only a few distant exceptions), in contrast to the more scattered pattern observed for alcoholic patients.

Treatment leads to altered abundance in certain major phyla and genera. Bar charts in Fig. 4A and B display the relative abundance of gut microbiota at the phylum level, while samples are clustered according to unweighted and weighted Unifrac. The results demonstrated that Firmicutes and Proteobacteria constitute the majority of the gut microbiota. In general, a decrease in the abundance of Firmicutes was observed in the patients following treatment. The trend was more apparent in non-alcoholic patients, with alcoholic patients demonstrating unaltered or even increased numbers, such as patients 12 and 14. In contrast, Proteobacteria, which constituted the second largest phylum in the gut microbiota, exhibited a divergent trend, with its abundance increasing post-treatment in 7 out of the 14 patients. The remaining half of the patients demonstrated unaltered or decreased abundance of Proteobacteria. No correlation was detected between the two different treatment groups.

A clustering tree is a method that clusters the samples based on the unweighted and weighted Unifracs. In the clustering tree, the closer two samples are located, the more similar their microbiota compositions are. By examining the clustering trees using unweighted and weighted Unifracs to showcase the microbiota similarities between the patients prior to and after the treatment, a much shorter distance was observed in each patient prior to and following treatment in the weighted tree as represented by the number of connection lines between bars in Fig. 4A and B. This indicates reduced pairwise disparity pre- and post-treatment due to the relative abundances of bacterial species considered as weights. When these weights (relative species abundances) are taken into account, the calculated weighted Unifracs are closer to each other, leading to shortened distances. For instance, patient 13 presented a greater unweighted UniFrac distance in comparison with the weighted distance.

Clustering analysis at the genus level for each sample was also performed (Fig. 4C). By comparing the abundance prior to and following treatment for each patient, a predominant reduction in Clostridium abundance was observed post-treatment, with a concurrent increase in Lactobacillus and decrease in Streptococcus and Faecalibacterium abundances in a small fraction of the patients. Particularly, reduction in one Streptococcus species was detected in only 2 patients post-treatment, which belonged to the non-alcoholic and combinatorial treatment with rifaximin plus probiotics groups. Bacteroides demonstrated a divergent trend, with certain patients presenting higher abundance of Bacteroides post-treatment, such as patient 1 , and certain others having decreased abundance, such as patient 2 . However, no correlation was observed between the change patterns and grouping criteria.

\section{Discussion}

The present study provided an insight into the varying response of alcoholic and non-alcoholic MHE patients to different treatments, including rifaximin alone or rifaximin plus probiotics, in terms of the gut microbiota composition. The current results demonstrated an overall decline in gut microbiota diversity following treatment, which was more apparent in MHE patients treated with rifaximin and probiotics. In addition, non-alcoholic MHE patients responded better, presenting a decreased microbiota diversity and ammonia-producing bacteria abundance, compared with alcoholic patients.

Gut microbiota is critical in maintaining normal intestinal functions, including digestion, absorption, nutrition supply and immune activation (16-19). Distinctive gut microbiota alterations are connected with the cognitive and inflammatory status in HE patients with liver cirrhosis $(20,21)$. A thorough understanding of their roles in the pathogenesis of $\mathrm{HE}$, particularly in MHE, is critical in the identification of appropriate strategies targeting the microbial species in order to restore the normal microbiota composition and functions. Given that gut microbiota is diverse in different populations, studies targeting a specific population are required in order to more precisely decipher the pathogenesis and identify therapeutic strategies. The southwestern Yunnan Province in China hosts a large population of hepatitis B and C patients, giving rise to increased number of MHE patients. Therefore, investigation into gut microbiota alterations in these patients may reveal distinct mechanisms of the disease pathogenesis and facilitate treatment strategy development specifically for this population.

Certain treatment regimens have been designed for the therapy of MHE, including administration of rifaximin (a semisynthetic antibiotic), probiotics, lactulose, prebiotics 


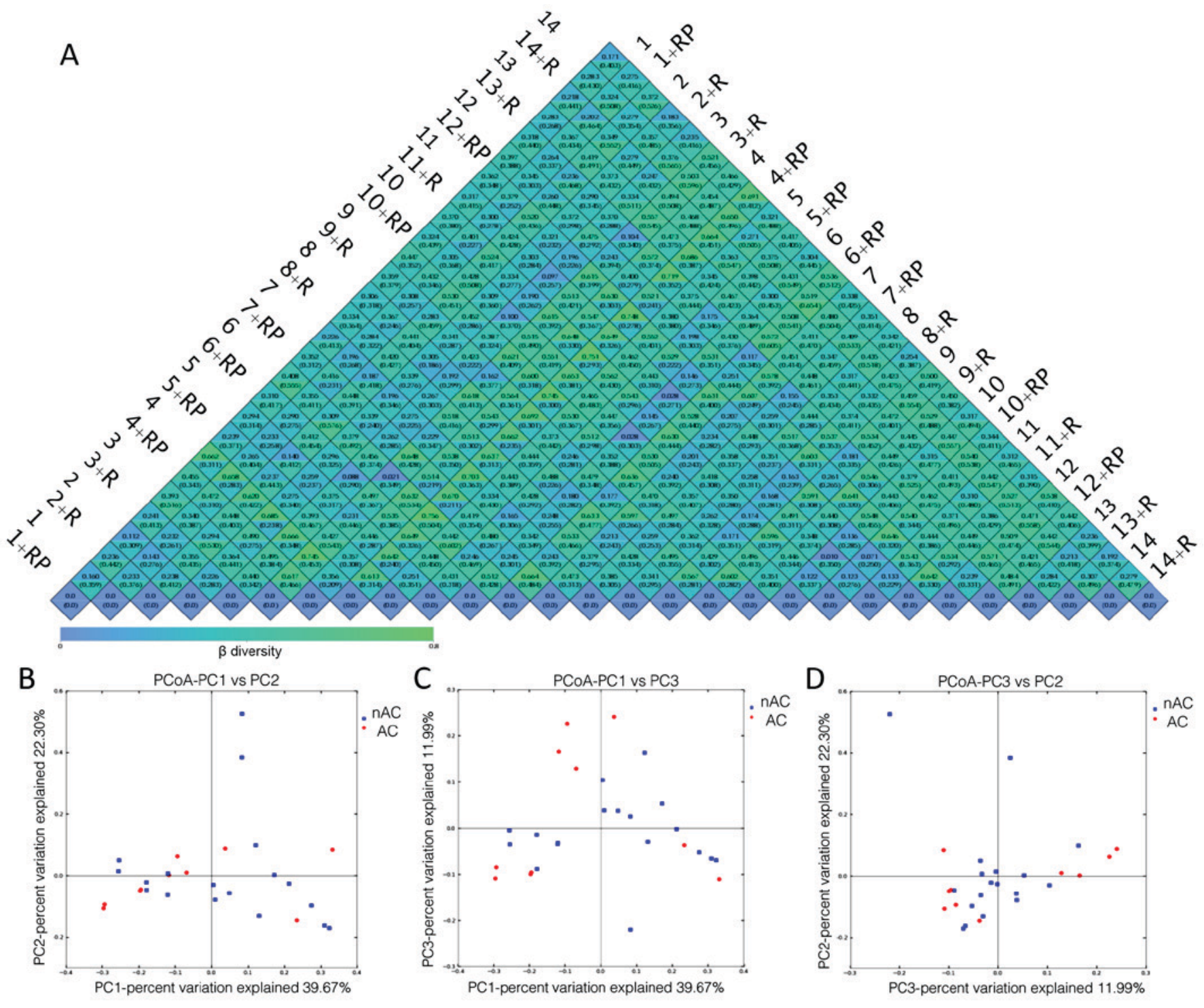

Figure 3. Alcohol addiction compromises treatment efficacy. (A) Heatmap demonstrating the weighted (top value in each box) and unweighted (bottom value in each box) Unifrac values between two patients in a pairwise comparison, prior to or following treatment, as determined by $\beta$ diversity analysis. (B) PcoA analysis of non-alcoholic and alcoholic patients in two principal coordinates (PC1 and PC2) using weighted Unifrac values. (C) PcoA analysis of non-alcoholic and alcoholic patients in two principal coordinates (PC1 and PC3) using weighted Unifrac values. (D) PcoA analysis of non-alcoholic and alcoholic patients in two principal coordinates (PC3 and PC2) using weighted Unifrac values.

and synbiotics (22). The study by Bajaj et al (22) demonstrated no significant microbiota alteration subsequent to rifaximin treatment, with the exception of a modest decrease in Veillonellaceae and increase in Eubacteriaceae. However, rifaximin administration contributed to cognitive functions by shifting the networks centered on Enterobacteriaceae, Porphyromonadaceae and Bacteroidaceae from pathogenic to beneficial metabolite linkages. Despite all these advances, there is a lack of mechanistic investigations on combination treatments in terms of their impact on gut microbiota. Therefore, the present study aimed to reveal the gut microbiota alterations in MHE patients treated with rifaximin plus probiotics, as compared with rifaximin alone. Further insight into how alcoholic and non-alcoholic MHE patients may respond to these two regimens was also examined.

In the current study, a general decline in gut microbiota diversity was observed when the MHE patients were treated with rifaximin alone or rifaximin plus probiotics. The difference in microbiota composition was also signified by the paired UniFrac of each MHE patient prior and subsequent to treatment in $\beta$ diversity analysis. This can be explained by the nature of rifaximin, which is an antibiotic intended to kill certain microbes, such as E. coli, thereby reducing diversity. MHE patients treated with rifaximin plus probiotics yielded an overall lower magnitude of decrease in the estimated species number following the treatment as compared with the pre-treatment value. Probiotics, which are microorganisms considered to be beneficial for a more balanced microorganism distribution in the gut when consumed, may account for this disparity (23). However, these patients demonstrated a more significant reduction in Shannon index following treatment. As Shannon index considers the relative abundance of bacterial species, this phenomenon suggests that the combined treatment of rifaximin plus probiotics may more significantly distort the relatively balanced distribution of microbial species abundance rather than reduce the number of bacterial species. Furthermore, the 


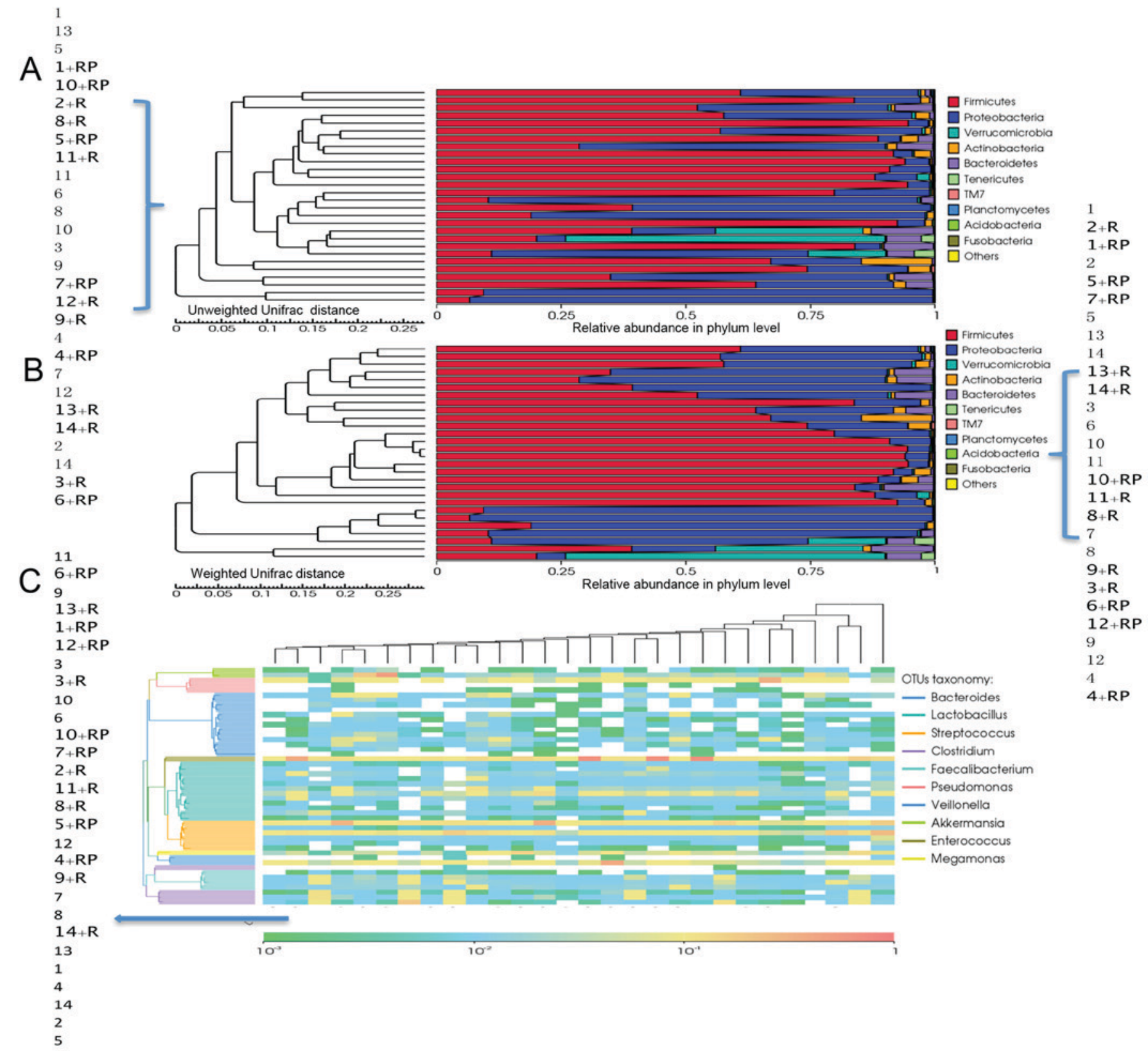

Figure 4. Treatment leads to altered abundance in certain major phyla and genera. The relative abundances of gut microbiota at the phylum level and clustering of the samples are presented, using (A) unweighted (amplified sample list to the left) and (B) weighted Unifrac values (amplified sample to the right). (C) Clustering analysis at the genus level for each sample [amplified sample list to the left, with sample names transposed (top name denotes the left most sample, whereas the bottom name denotes the right most sample)].

presence of probiotics improves the symptoms by shifting the microbiota composition from pathological to beneficial distribution, thereby creating a more favorable gut environment to restore the beneficial species and microbiota functions that may be partially compromised by rifaximin. However, $\beta$ analysis did not reveal a considerable difference in the unweighted or weighted Unifrac value of each MHE patient between the two treatment groups. The Unifrac values were calculated based on the phylogenetic tree, or the relative position of each bacterial species in the evolutionary tree. This suggests that, despite the impact of probiotics on microbiota diversity, this impact may be negligible when considering the entire phylogenetic tree.

Upon comparison of alcoholic and non-alcoholic MHE patients in the present study, non-alcoholic subjects presented a predominant reduction in Shannon diversity index and higher pairwise Unifrac values post treatment vs. pre-treatment values, when compared to alcoholic MHE patients. Non-alcoholic patients also presented a more consistent trend in the abundance of certain major bacterial phyla post-treatment, such as decline in Firmicutes. Given the much lower abundance of Firmicutes in healthy individuals in Yunnan (24), it was suggested that alcoholic MHE patients possess lower capability in the restoration of gut microbiota and have a reduced response to the treatment regimen. Similarly, alcoholic MHE patients presented more scattered weighted PcoA results, suggesting that their response to treatment may be more unpredictable. Furthermore, certain non-alcoholic MHE patients demonstrated a decrease post-treatment in 
Streptococcus, a urease-producing bacteria genus, while alcoholic patients did not exhibit this change. The decrease in Streptococcus may consequently lower ammonia levels and improve patient conditions.

In the present study, clustering analysis by weighted and unweighted Unifrac distances produced two distinct clusters, with shorter weighted Unifrac distances observed in the majority of the MHE patients pre- and post-treatment. As weighted Unifrac values take into consideration the relative abundance of each phylum, these shorter distances suggest a relatively small change in the abundances of major phyla. By contrast, as unweighted Unifrac values consider all existing phyla regardless of their abundance, the longer distances of each patient pre- and post-treatment in the clusters indicate a marked change in the composition or the number of microorganisms at the phylum level. Nevertheless, this does not exclude the differences in the abundances of major phyla, however subtle they are. Certain patients even demonstrated considerable alterations in the composition of these bacteria, such as patient ZFY3. In general, the abundance of Firmicutes declined following treatment, particularly in non-alcoholic MHE patients.

Ammonia produced by gut microbes is regarded as an important inducing agent of MHE, and its level is highly correlated with MHE pathogenesis (25). Specific bacterial species carry urease-encoding genes and have been found to be associated with ammonia metabolism, including Clostridium, Klebsiella, Proteus, Veillonella and Helicobacter $(8,11)$. Zhang et al (26) identified that Streptococcaceae and Veillonellaceae are enriched in liver cirrhotic patients with or without MHE, and MHE-unique interplay pattern of gut microbiota is greatly influenced by these two bacterial families. Bajaj et al (22) also noted no significant gut microbiota alteration following rifaximin treatment, with an exception of a modest decrease in Veillonellaceae and an increase in Eubacteriaceae. However, the present study did not demonstrate any evident alterations in the two genera of Streptococcus and Veillonella post-treatment, with the exception of two non-alcoholic MHE patients treated with rifaximin plus probiotics, who presented decreased Streptococcus levels. The discrepancy may lie in the different taxonomic levels at which statistical analysis was performed, since the present study conducted analysis at the genus level, whereas the aforementioned analysis (22) was performed at the family level. Another explanation may be that the Veillonellaceae family is enriched in MHE, but its abundance is not significantly altered by rifaximin treatment.

The current investigation also detected a robust decline in the genus of Clostridium, which belongs to the Firmicutes phylum. Specific Clostridium species are considered to be hyper-ammonia producing, such as Clostridium aminophilum and Clostridium histolyticum. The proteases secreted by Clostridium histolyticum can digest native and denatured proteins into amino acids with the production of ammonia (27). Although Clostridium was not identified to be highly enriched in MHE patients in the present study its reduction post-treatment may lead to declined ammonia levels in the blood and thereby reduced severe ammonia toxicosis, thus contributing to improved cognitive conditions in the MHE patients. Besides, Clostridium, Streptococcus and Veillonella, which belong to the Firmicutes phylum and are ammonia-producing bacteria, contributed to the decline in Firmicutes at the phylum level, as observed in the current study. The decline in these bacterial genera post-treatment leads to partial restoration of the microbiota composition as compared with healthy individuals, and therefore improvement in clinical conditions (4).

Lactobacillus, which also belongs to the Firmicutes phylum, demonstrated an increase in certain patients following treatment in the present study. This may also beneficial for the treatment of MHE, since its metabolic product (lactic acid) decreases gut $\mathrm{pH}$ and thereby kills the bacterial species that have urease to convert nutrition into ammonia. Lactobacillus not only reduces ammonia levels in the gut, but also creates a favorable environment for the growth of probiotics, such as Lactobacillus and Bifidobacterium. Jointly, these changes contribute to the modulation of gut microbiota dysbiosis associated with MHE (8).

In conclusion, the present study investigated the effect of different treatment strategies, including rifaximin alone or rifaximin plus probiotics, on gut microbiota in $\mathrm{MHE}$ patients. The addition of probiotics in the treatment regimen distorted the distribution of bacteria in the gut and reduced Streptococcus abundance. In addition, non-alcoholic MHE patients presented a higher magnitude of gut microbiota alterations subsequent to treatment, particularly reduction in the abundance of Firmicutes.

\section{Acknowledgements}

The current study was financially supported by grants from the National Natural Science Foundation of China (grant no. 81260077). The authors would like to thank Professor Xiangyang Kong (Medical College of Kunming University of Science and Technology, Kunming, China), Professor Zhigang Zhang (Kunming Institute of Zoology, Chinese Academy of Sciences, Kunming, China) and Dr Junhong Su (Medical College of Kunming University of Science and Technology, Kunming, China) for their exceptional technical assistance and help with the manuscript.

\section{References}

1. Dbouk N and McGuire BM: Hepatic encephalopathy: A review of its pathophysiology and treatment. Curr Treat Options Gastroenterol 9: 464-474, 2006.

2. Kato A, Tanaka H, Kawaguchi T, Kanazawa H, Iwasa M, Sakaida I, Moriwaki H, Murawaki Y, Suzuki K and Okita K: Nutritional management contributes to improvement in minimal hepatic encephalopathy and quality of life in patients with liver cirrhosis: A preliminary, prospective, open-label study. Hepatol Res 43: 452-458, 2013

3. Amodio P, Montagnese S, Gatta A and Morgan MY: Characteristics of minimal hepatic encephalopathy. Metab Brain Dis 19: 253-267, 2004.

4. Bernardini P and Fischer JE: Amino acid imbalance and hepatic encephalopathy. Annu Rev Nutr 2: 419-454, 1982.

5. Norman $\mathrm{K}$ and Pirlich M: Gastrointestinal tract in liver disease: Which organ is sick? Curr Opin Clin Nutr Metab Care 11: 613-619, 2008.

6. Prakash R and Mullen KD: Mechanisms, diagnosis and management of hepatic encephalopathy. Nat Rev Gastroenterol Hepatol 7: 515-525, 2010.

7. Kawaguchi T, Taniguchi E and Sata M: Effects of oral branched-chain amino acids on hepatic encephalopathy and outcome in patients with liver cirrhosis. Nutr Clin Pract 28: 580-588, 2013. 
8. Riordan SM and Williams R: Gut flora and hepatic encephalopathy in patients with cirrhosis. N Engl J Med 362: 1140-1142, 2010.

9. Kawaguchi T, Taniguchi E and Sata M: Motor vehicle accidents: How should cirrhotic patients be managed? World J Gastroenterol 18: 2597-2599, 2012.

10. Kawaguchi T, Suetsugu T, Ogata S, Imanaga M, Ishii K, Esaki N, Sugimoto M, Otsuyama J, Nagamatsu A, Taniguchi E, et al: An association between dietary habits and traffic accidents in patients with chronic liver disease: A data-mining analysis Biomed Rep 4: 615-622, 2016.

11. Bass NM, Mullen KD, Sanyal A, Poordad F, Neff G, Leevy CB Sigal S, Sheikh MY, Beavers K, Frederick T, et al: Rifaximin treatment in hepatic encephalopathy. N Engl J Med 362: $1071-1081,2010$

12. Zhan T and Stremmel W: The diagnosis and treatment of minimal hepatic encephalopathy. Dtsch Arztebl Int 109: 180-187, 2012.

13. Kumar MS, Kaur G and Sandhu AK: Genomic DNA isolation from fungi, algae, plant, bacteria and human blood using CTAB. Int J Sci Res 3: 617-618, 2014.

14. Qin J, Li R, Raes J, Arumugam M, Burgdorf KS, Manichanh C, Nielsen T, Pons N, Levenez F, Yamada T, et al: A human gut microbial gene catalogue established by metagenomic sequencing. Nature 464: 59-65, 2010.

15. Kindt R and Coe R: Tree diversity analysis: A manual and software for common statistical methods for ecological and biodiversity studies. World Agroforestry Centre, 2005.

16. Khoruts A and Sadowsky MJ: Therapeutic transplantation of the distal gut microbiota. Mucosal Immunol 4: 4-7, 2011.

17. Wikoff WR, Anfora AT, Liu J, Schultz PG, Lesley SA, Peters EC and Siuzdak G: Metabolomics analysis reveals large effects of gut microflora on mammalian blood metabolites. Proc Natl Acad Sci USA 106: 3698-3703, 2009.

18. MacDonald TT, Monteleone I, Fantini MC and Monteleone G: Regulation of homeostasis and inflammation in the intestine. Gastroenterology 140: 1768-1775, 2011.
19. Cho I and Blaser MJ: The human microbiome: At the interface of health and disease. Nat Rev Genet 13: 260-270, 2012.

20. Bajaj JS, Hylemon PB, Ridlon JM, Heuman DM, Daita K, White MB, Monteith P, Noble NA, Sikaroodi M and Gillevet PM: Colonic mucosal microbiome differs from stool microbiome in cirrhosis and hepatic encephalopathy and is linked to cognition and inflammation. Am J Physiol Gastrointest Liver Physiol 303: G675-G685, 2012

21. Bajaj JS, Ridlon JM, Hylemon PB, Thacker LR, Heuman DM, Smith S, Sikaroodi M and Gillevet PM: Linkage of gut microbiome with cognition in hepatic encephalopathy. Am J Physiol Gastrointest Liver Physiol 302: G168-G175, 2012.

22. Bajaj JS, Heuman DM, Sanyal AJ, Hylemon PB, Sterling RK, Stravitz RT, Fuchs M, Ridlon JM, Daita K, Monteith P, et al: Modulation of the metabiome by rifaximin in patients with cirrhosis and minimal hepatic encephalopathy. PLoS One 8: e60042, 2013.

23. Rijkers GT, de Vos WM, Brummer RJ, Morelli L, Corthier G and Marteau P: Health benefits and health claims of probiotics: Bridging science and marketing. Br J Nutr 106: 1291-1296, 2011.

24. Kuang YS, Li SH, Guo Y, Lu JH, He JR, Luo BJ, Jiang FJ, Shen H, Papasian CJ, Pang H, et al: Composition of gut microbiota in infants in China and global comparison. Sci Rep 6: 36666, 2016.

25. Jain L, Sharma BC, Srivastava S, Puri SK, Sharma P and Sarin S: Serum endotoxin, inflammatory mediators, and magnetic resonance spectroscopy before and after treatment in patients with minimal hepatic encephalopathy. J Gastroenterol Hepatol 28: $1187-1193,2013$

26. Zhang Z, Zhai H, Geng J, Yu R, Ren H, Fan H and Shi P: Large-scale survey of gut microbiota associated with MHE Via 16S rRNA-based pyrosequencing. Am J Gastroenterol 108: 1601-1611, 2013.

27. Oakley CL and Warrack GH: The alpha, beta and gamma antigens of Clostridium histolyticum (Weinberg \& Séguin, 1916). J Gen Microbiol 4: 365-373, 1950 